\title{
Pacific
}

Journal of

Mathematics

\section{WHITNEY UMBRELLAS AND SWALLOWTAILS}

TAKASHI NISHIMURA

Volume 252 No. 2

August 2011 


\title{
WHITNEY UMBRELLAS AND SWALLOWTAILS
}

\author{
TAKASHI NISHIMURA
}

Dedicated to Professor Shyuichi Izumiya on the occasion of his sixtieth birthday

\begin{abstract}
We introduce map germs of pedal unfolding type and the notion of normalized Legendrian map germs. We show that the fundamental theorem of calculus provides a natural one-to-one correspondence between Whitney umbrellas of pedal unfolding type and normalized swallowtails.
\end{abstract}

\section{Introduction}

The map germ

$$
f(x, y)=\left(x y, x^{2}, y\right)
$$

is known as the normal form of Whitney umbrella, after Whitney's pioneering works $[1943 ; 1944]$. Compose the germ (1) with the coordinate transformations

$$
h_{s}(x, y)=\left(x, x^{2}+y\right) \quad \text { and } \quad h_{t}(X, Y, Z)=(X,-Z,-Y+Z),
$$

where $(X, Y, Z)$ are the standard coordinates of the target space $\mathbb{R}^{3}$. This leads to the map germ

$$
g(x, y)=h_{t} \circ f \circ h_{s}(x, y)=\left(x^{3}+x y,-x^{2}-y, y\right) .
$$

Set

$$
\begin{aligned}
G(x, y) & =\left(\int_{0}^{x}\left(x^{3}+x y\right) d x, \int_{0}^{x}\left(-x^{2}-y\right) d x, y\right) \\
& =\left(\frac{1}{4} x^{4}+\frac{1}{2} x^{2} y,-\frac{1}{3} x^{3}-x y, y\right) .
\end{aligned}
$$

Compose the map germ (3) with the scaling transformations

$$
H_{s}(x, y)=\left(x, \frac{1}{6} y\right) \quad \text { and } \quad H_{t}(X, Y, Z)=(12 X, 12 Y, 6 Z)
$$

to obtain the map germ

$$
H_{t} \circ G \circ H_{s}(x, y)=\left(3 x^{4}+x^{2} y,-4 x^{3}-2 x y, y\right),
$$

MSC2010: 53A05, 57R45, 58K25.

Keywords: Whitney umbrella, pedal unfolding type, normalized swallowtail, swallowtail. 
known as the normal form of the swallowtail [Bruce and Giblin 1992, page 129].

Two $C^{\infty}$ map germs $\varphi, \psi:\left(\mathbb{R}^{2}, 0\right) \rightarrow\left(\mathbb{R}^{3}, 0\right)$ are said to be $\mathscr{A}$-equivalent if there exist germs of $C^{\infty}$ diffeomorphisms

$$
h_{s}:\left(\mathbb{R}^{2}, 0\right) \rightarrow\left(\mathbb{R}^{2}, 0\right) \quad \text { and } \quad h_{t}:\left(\mathbb{R}^{3}, 0\right) \rightarrow\left(\mathbb{R}^{3}, 0\right),
$$

such that $\psi=h_{t} \circ \varphi \circ h_{s}$. A $C^{\infty}$ map germ $\varphi:\left(\mathbb{R}^{2}, 0\right) \rightarrow\left(\mathbb{R}^{3}, 0\right)$ is called a Whitney umbrella if it is $\mathscr{A}$-equivalent to (1); it is called a swallowtail if it is $\mathscr{A}$-equivalent to (4). As seen above, the Whitney umbrella (1) produces the swallowtail (4) via (2) and (3). By the converse procedure, the swallowtail (4) produces the Whitney umbrella (1).

It is impossible to produce a swallowtail by integrating (1) directly. This is because the discriminant set of (4) is not diffeomorphic to the discriminant set of

$$
(x, y) \mapsto\left(\int_{0}^{x} x y d x, \int_{0}^{x} x^{2} d x, y\right) .
$$

Note that the form (2) may be written as follows:

$$
g(x, y)=\left(x\left(x^{2}+y\right),-\left(x^{2}+y\right), y\right)=\left(b\left(-x,-\left(x^{2}+y\right)\right), y\right),
$$

where $b(X, Y)=(X Y, Y)$ ( $b$ stands for "blowdown").

Definition 1.1. (i) A $C^{\infty}$ map germ $\varphi:\left(\mathbb{R}^{2}, 0\right) \rightarrow\left(\mathbb{R}^{3}, 0\right)$ having the following form is said to be of pedal unfolding type.

$$
\varphi(x, y)=(n(x, y) p(x, y), p(x, y), y)=(b(n(x, y), p(x, y)), y),
$$

where $n:\left(\mathbb{R}^{2}, 0\right) \rightarrow(\mathbb{R}, 0)$ is a $C^{\infty}$ function germ, such that

$$
\frac{\partial n}{\partial x}(0,0) \neq 0 \quad \text { and } \quad p:\left(\mathbb{R}^{2}, 0\right) \rightarrow(\mathbb{R}, 0) \text { is a } C^{\infty} \text { function germ. }
$$

(ii) For a $C^{\infty}$ map germ of pedal unfolding type

$$
\varphi(x, y)=(n(x, y) p(x, y), p(x, y), y),
$$

set

$$
\mathscr{I}(\varphi)(x, y)=\left(\int_{0}^{x} n(x, y) p(x, y) d x, \int_{0}^{x} p(x, y) d x, y\right) .
$$

The map germ $\Phi(\varphi):\left(\mathbb{R}^{2}, 0\right) \rightarrow\left(\mathbb{R}^{3}, 0\right)$ is called the integration of $\varphi$.

(iii) A $C^{\infty}$ map germ $\Phi:\left(\mathbb{R}^{m}, 0\right) \rightarrow\left(\mathbb{R}^{m+1}, 0\right)$ is called a Legendrian map germ if there exists a germ of $C^{\infty}$ vector field $v_{\Phi}:\left(\mathbb{R}^{m}, 0\right) \rightarrow T_{1} \mathbb{R}^{m+1}$ along $\Phi$ such that

$$
\frac{\partial \Phi}{\partial x_{1}}\left(x_{1}, \ldots, x_{m}\right) \cdot v_{\Phi}\left(x_{1}, \ldots, x_{m}\right)=\cdots=\frac{\partial \Phi}{\partial x_{m}}\left(x_{1}, \ldots, x_{m}\right) \cdot v_{\Phi}\left(x_{1}, \ldots, x_{m}\right)=0
$$


and the map germ $L_{\Phi}:\left(\mathbb{R}^{m}, 0\right) \rightarrow T_{1} \mathbb{R}^{m+1}$ defined by

$$
L_{\Phi}\left(x_{1}, \ldots, x_{m}\right)=\left(\Phi\left(x_{1}, \ldots, x_{m}\right), v_{\Phi}\left(x_{1}, \ldots, x_{m}\right)\right)
$$

is nonsingular. $L_{\Phi}$ is called a Legendrian lift of $\Phi$. (Here the dot stands for the scalar product of two vectors of $T_{\Phi(x, y)} \mathbb{R}^{m+1}$, and $T_{1} \mathbb{R}^{m+1}$ is the unit tangent bundle of $\mathbb{R}^{m+1}$.) The $C^{\infty}$ vector field $v_{\Phi}$ is called a unit normal vector field of $\Phi$.

(iv) A Legendrian map germ $\Phi:\left(\mathbb{R}^{2}, 0\right) \rightarrow\left(\mathbb{R}^{3}, 0\right)$ is said to be normalized if it has the form

$$
\Phi(x, y)=\left(\Phi_{1}(x, y), \Phi_{2}(x, y), y\right)
$$

with

$$
\frac{\partial \Phi_{2}}{\partial x}(0,0)=0
$$

and if, furthermore,

$$
v_{\Phi}(0,0)=\frac{\partial}{\partial X} \quad \text { or } \quad v_{\Phi}(0,0)=-\frac{\partial}{\partial X} .
$$

(v) For a normalized Legendrian map germ $\Phi(x, y)=\left(\Phi_{1}(x, y), \Phi_{2}(x, y), y\right)$, set

$$
\mathscr{D}(\Phi)(x, y)=\left(\frac{\partial \Phi_{1}}{\partial x}(x, y), \frac{\partial \Phi_{2}}{\partial x}(x, y), y\right) .
$$

The map germ $\mathscr{D}(\Phi):\left(\mathbb{R}^{2}, 0\right) \rightarrow\left(\mathbb{R}^{3}, 0\right)$ is called the differential of $\Phi$.

We showed in [Nishimura 2010] that any germ $\varphi:\left(\mathbb{R}^{2}, 0\right) \rightarrow\left(\mathbb{R}^{3}, 0\right)$ of a oneparameter pedal unfolding of a spherical pedal curve has the form (6). Hence, a map germ $\varphi$ having the form (6) is said to be of pedal unfolding type. As shown in [Nishimura 2010], not only nonsingular map germs, but also Whitney umbrellas may be realized as germs of one-parameter pedal unfoldings of spherical pedal curves. For more information on Legendrian map germs, see [Arnold et al. 1985; Izumiya 1987; Zakalyukin 1976; 1983]. Note that both (3) and (10) are normalized Legendrian map germs.

Proposition 1.2. (i) If $\varphi:\left(\mathbb{R}^{2}, 0\right) \rightarrow\left(\mathbb{R}^{3}, 0\right)$ is a $C^{\infty}$ map germ of pedal unfolding type, $\mathscr{}(\varphi)$ is a normalized Legendrian map germ.

(ii) If $\Phi:\left(\mathbb{R}^{2}, 0\right) \rightarrow\left(\mathbb{R}^{3}, 0\right)$ is a normalized Legendrian map germ, $\mathscr{D}(\Phi)$ is a map germ of pedal unfolding type.

Set

$\mathscr{W}=\left\{\varphi:\left(\mathbb{R}^{2}, 0\right) \rightarrow\left(\mathbb{R}^{3}, 0\right)\right.$ Whitney umbrella of pedal unfolding type $\}$, $\mathscr{S}=\left\{\Phi:\left(\mathbb{R}^{2}, 0\right) \rightarrow\left(\mathbb{R}^{3}, 0\right)\right.$ normalized swallowtail $\}$. 
The main purpose of this paper is to show the following:

Theorem 1.3. (i) The map $\mathscr{I}: \mathcal{W} \rightarrow \mathscr{S}$ defined by $\mathscr{W} \ni \varphi \mapsto \mathscr{I}(\varphi) \in \mathscr{Y}$ is welldefined and bijective.

(ii) The map $\mathscr{D}: \mathscr{Y} \rightarrow \mathscr{W}$ defined by $\mathscr{Y} \ni \Phi \mapsto \mathscr{D}(\Phi) \in \mathscr{W}$ is well-defined and bijective.

Incidentally, we show Theorem 1.4. A $C^{\infty}$ map germ $\Phi:\left(\mathbb{R}^{2}, 0\right) \rightarrow\left(\mathbb{R}^{3}, 0\right)$ is called a cuspidal edge if $\Phi$ is $\mathscr{A}$-equivalent to the following:

$$
(x, y) \mapsto\left(\frac{1}{3} x^{3}, \frac{1}{2} x^{2}, y\right) .
$$

Set

$$
\begin{aligned}
& \mathcal{N}=\left\{\varphi:\left(\mathbb{R}^{2}, 0\right) \rightarrow\left(\mathbb{R}^{3}, 0\right) \text { nonsingular map germ of pedal unfolding type }\right\}, \\
& \mathscr{C}=\left\{\Phi:\left(\mathbb{R}^{2}, 0\right) \rightarrow\left(\mathbb{R}^{3}, 0\right) \text { normalized cuspidal edge }\right\} .
\end{aligned}
$$

Theorem 1.4. (i) The map $\mathscr{I}: \mathcal{N} \rightarrow \mathscr{C}$ defined by $\mathcal{N} \ni \varphi \mapsto \Phi(\varphi) \in \mathscr{C}$ is welldefined and bijective.

(ii) The map $\mathscr{D}: \mathscr{C} \rightarrow \mathcal{N}$ defined by $\mathscr{C} \ni \Phi \mapsto \mathscr{D}(\Phi) \in \mathcal{N}$ is well-defined and bijective.

Any stable map germ $\left(\mathbb{R}^{2}, 0\right) \rightarrow\left(\mathbb{R}^{3}, 0\right)$ is either a Whitney umbrella or nonsingular, and any Legendrian stable singularity $\left(\mathbb{R}^{2}, 0\right) \rightarrow\left(\mathbb{R}^{3}, 0\right)$ is either a cuspidal edge or a swallowtail (see [Arnold et al. 1985], for example). Theorems 1.3 and 1.4 can thus be regarded as a "fundamental theorem of calculus" for stable map germs $\left(\mathbb{R}^{2}, 0\right) \rightarrow\left(\mathbb{R}^{3}, 0\right)$ and Legendrian stable singularities $\left(\mathbb{R}^{2}, 0\right) \rightarrow\left(\mathbb{R}^{3}, 0\right)$.

Based on Theorems 1.3 and 1.4, it is natural to ask:

Question 1.5. (i) Let $\varphi_{1}, \varphi_{2}:\left(\mathbb{R}^{2}, 0\right) \rightarrow\left(\mathbb{R}^{3}, 0\right)$ be two $C^{\infty}$ map germs of pedal unfolding type. Suppose that $\varphi_{1}$ is $\mathscr{A}$-equivalent to $\varphi_{2}$. Is $\mathscr{I}\left(\varphi_{1}\right)$ necessarily $\mathscr{A}$-equivalent to $\mathscr{S}\left(\varphi_{2}\right)$ ?

(ii) Let $\Phi_{1}, \Phi_{2}:\left(\mathbb{R}^{2}, 0\right) \rightarrow\left(\mathbb{R}^{3}, 0\right)$ be two normalized Legendrian map germs. Suppose that $\Phi_{1}$ is $\mathscr{A}$-equivalent to $\Phi_{2}$. Is $\mathscr{D}\left(\Phi_{1}\right)$ necessarily $\mathscr{A}$-equivalent to $\mathscr{D}\left(\Phi_{2}\right)$ ?

In Section 2, several preparations for the proofs of Theorems 1.3 and 1.4 and the proof of Proposition 1.2 are given. Theorems 1.3 and 1.4 are proved in Section 3 and Section 4 respectively.

\section{Preliminaries}

Function germs with two variables and map germs with two variables. Let $\mathscr{E}_{2}$ be the set of $C^{\infty}$ function germs $\left(\mathbb{R}^{2}, 0\right) \rightarrow \mathbb{R}$, and let $m_{2}$ be the subset of $\mathscr{E}_{2}$ 
consisting of $C^{\infty}$ function germs $\left(\mathbb{R}^{2}, 0\right) \rightarrow(\mathbb{R}, 0)$. The set $\mathscr{E}_{2}$ has a natural $\mathbb{R}$ algebra structure. For a $C^{\infty}$ map germ $\varphi:\left(\mathbb{R}^{2}, 0\right) \rightarrow\left(\mathbb{R}^{2}, 0\right)$, let $\varphi^{*}: \mathscr{E}_{2} \rightarrow \mathscr{E}_{2}$ be the $\mathbb{R}$-algebra homomorphism defined by $\varphi^{*}(u)=u \circ \varphi$. Set $Q(\varphi)=\mathscr{E}_{2} / \varphi^{*} m_{2} \mathscr{E}_{2}$. Then, $Q(\varphi)$ is an $\mathbb{R}$-algebra. A special case of [Mather 1969, Theorem 2.1] follows.

Proposition 2.1. Let $p:\left(\mathbb{R}^{2}, 0\right) \rightarrow(\mathbb{R}, 0)$ be a $C^{\infty}$ function germ.

(i) The $\mathbb{R}$-algebra $Q(p(x, y), y)$ is isomorphic to $Q\left(x^{2}, y\right)$ if and only if

$$
\frac{\partial p}{\partial x}(0,0)=0 \quad \text { and } \quad \frac{\partial^{2} p}{\partial x^{2}}(0,0) \neq 0 .
$$

(ii) The $\mathbb{R}$-algebra $Q(p(x, y), y)$ is isomorphic to $Q(x, y)$ if and only if

$$
(x, y) \mapsto(p(x, y), y)
$$

is a germ of $C^{\infty}$ diffeomorphism.

Definition 2.2 [Mond 1985]. Let $T: \mathbb{R}^{2} \rightarrow \mathbb{R}^{2}$ be the linear transformation of the form $T(s, \lambda)=(-s, \lambda)$. Two $C^{\infty}$ function germs $p_{1}, p_{2}:\left(\mathbb{R}^{2}, 0\right) \rightarrow(\mathbb{R}, 0)$ are said to be $\mathscr{K}^{T}$-equivalent if there exists a germ of $C^{\infty}$ diffeomorphism

$$
h:\left(\mathbb{R}^{2}, 0\right) \rightarrow\left(\mathbb{R}^{2}, 0\right)
$$

of the form $h \circ T=T \circ h$, and a $C^{\infty}$ function germ $M:\left(\mathbb{R}^{2},(0,0)\right) \rightarrow \mathbb{R}-\{0\}$ of the form $M \circ T=M$, such that $p_{1} \circ h(x, y)=M(x, y) p_{2}(x, y)$.

Theorem 2.3 [Mond 1985]. Two $C^{\infty}$ map germs $\varphi_{i}:\left(\mathbb{R}^{2}, 0\right) \rightarrow\left(\mathbb{R}^{3}, 0\right)(i=1,2)$ of the form

$$
\varphi_{i}(x, y)=\left(x p_{i}\left(x^{2}, y\right), x^{2}, y\right), \quad \text { where } p_{i}\left(x^{2}, y\right) \notin m_{2}^{\infty} \quad(i=1,2)
$$

are A-equivalent if and only if the function germs $p_{i}\left(x^{2}, y\right)$ are $\mathscr{K}^{T}$-equivalent. Here,

$$
m_{2}^{\infty}=\left\{q:\left(\mathbb{R}^{2}, 0\right) \rightarrow(\mathbb{R}, 0) \mid \frac{\partial^{i+j} q}{\partial x^{i} \partial y^{j}}(0,0)=0 \text { for all } i, j \in\{0\} \cup \mathbb{N}\right\} .
$$

From this and the Malgrange preparation theorem [Arnold et al. 1985], we have:

Corollary 2.4. Two $C^{\infty}$ map germs $\varphi_{i}:\left(\mathbb{R}^{2}, 0\right) \rightarrow\left(\mathbb{R}^{3}, 0\right)(i=1,2)$ of the form

$$
\varphi_{i}(x, y)=\left(n_{i}(x, y) p_{i}\left(x^{2}, y\right), x^{2}, y\right),
$$

where $p_{i}\left(x^{2}, y\right) \notin m_{2}^{\infty}$ and $\left(\partial n_{i} / \partial x\right)(0,0) \neq 0$ for $i=1,2$, are A-equivalent if and only if the function germs $p_{i}\left(x^{2}, y\right)$ are $\mathscr{K}^{T}$-equivalent. 
Map germs of pedal unfolding type. Let $\varphi: I \times J \rightarrow \mathbb{R}^{3}$ be a representative of a given $C^{\infty}$ map germ of pedal unfolding type, where $I, J$ are sufficiently small intervals containing the origin of $\mathbb{R}$. We may put $\varphi(x, y)=(n(x, y) p(x, y), p(x, y))$. Set

$$
\Phi(x, y)=\left(\Phi_{1}(x, y), \Phi_{2}(x, y), y\right)=\left(\int_{0}^{x} n(x, y) p(x, y) d x, \int_{0}^{x} p(x, y) d x, y\right)
$$

and

$$
\tilde{\mu}_{\Phi}(x, y)=\frac{\partial}{\partial X}-n(x, y) \frac{\partial}{\partial Y} .
$$

Since $\tilde{\mu}_{\Phi}(x, y) \neq 0$ for any $x \in I$ and $y \in J$, for any fixed $y \in J$ we may define the map germ $L_{\Phi, y}:(\mathbb{R}, 0) \rightarrow T_{1} \mathbb{R}^{2}$ as

$$
L_{\Phi, y}(x)=\left(\left(\Phi_{1}(x, y), \Phi_{2}(x, y)\right), \frac{\tilde{\mu}_{\Phi}(x, y)}{\left\|\tilde{\mu}_{\Phi}(x, y)\right\|}\right),
$$

where $T_{1} \mathbb{R}^{2}$ is the unit tangent bundle of $\mathbb{R}^{2}$. Then, since $\varphi$ is a representative of a map germ of pedal unfolding type, we have:

Lemma 2.5. For any $y \in J, L_{\Phi, y}:(\mathbb{R}, 0) \rightarrow T_{1} \mathbb{R}^{2}$ is a Legendrian lift of the map germ $x \mapsto\left(\Phi_{1}(x, y), \Phi_{2}(x, y)\right)$.

This implies:

Lemma 2.6. For any $y \in J$, the map germ $\widetilde{\Phi}_{y}:(\mathbb{R}, 0) \rightarrow\left(\mathbb{R}^{2}, 0\right)$ defined by $\widetilde{\Phi}_{y}(x)=\left(\Phi_{1}(x, y), \Phi_{2}(x, y)\right)$ is a Legendrian map germ.

Next, set

$$
\tilde{v}_{\Phi}(x, y)=\tilde{\mu}_{\Phi}(x, y)-\left(\frac{\partial \Phi_{1}}{\partial y}(x, y)-n(x, y) \frac{\partial \Phi_{2}}{\partial y}(x, y)\right) \frac{\partial}{\partial Z} .
$$

Lemma 2.7. For any $x \in I$ and $y \in J$,

$$
\tilde{v}_{\Phi}(x, y) \cdot \frac{\partial \Phi}{\partial x}(x, y)=0, \quad \tilde{v}_{\Phi}(x, y) \cdot \frac{\partial \Phi}{\partial y}(x, y)=0 .
$$

Since $\tilde{v}_{\Phi}(x, y) \neq 0$ for any $x \in I$ and $y \in J$, we may define the map germ

$$
L_{\Phi}:\left(\mathbb{R}^{2}, 0\right) \rightarrow T_{1} \mathbb{R}^{3}
$$

as

$$
L_{\Phi}(x, y)=\left(\Phi(x, y), \frac{\tilde{v}_{\Phi}(x, y)}{\left\|\tilde{v}_{\Phi}(x, y)\right\|}\right) .
$$

Then Lemma 2.7 implies successively:

Lemma 2.8. $L_{\Phi}:\left(\mathbb{R}^{2}, 0\right) \rightarrow T_{1} \mathbb{R}^{3}$ is a Legendrian lift of $\Phi$.

Lemma 2.9. $\Phi:\left(\mathbb{R}^{2}, 0\right) \rightarrow\left(\mathbb{R}^{3}, 0\right)$ is a Legendrian map germ. 
Normalized Legendrian map germs. Let $\Phi: U \rightarrow \mathbb{R}^{3}$ be a representative of a given normalized Legendrian map germ $\left(\mathbb{R}^{2}, 0\right) \rightarrow\left(\mathbb{R}^{3}, 0\right)$, where $U$ is a sufficiently small neighborhood of the origin of $\mathbb{R}^{2}$. We assume that the origin of $\mathbb{R}^{2}$ is a singular point of $\Phi$. By condition (7) of the definition of normalized Legendrian map germs, we may assume that $\Phi$ has the form

$$
\Phi(x, y)=\left(\Phi_{1}(x, y), \Phi_{2}(x, y), y\right) .
$$

Since $\Phi$ is a representative of a Legendrian map germ, we have the following:

Lemma 2.10. There exists a $C^{\infty}$ vector field $v_{\Phi}$ along $\Phi$,

$$
v_{\Phi}(x, y)=n_{1}(x, y) \frac{\partial}{\partial X}+n_{2}(x, y) \frac{\partial}{\partial Y}+n_{3}(x, y) \frac{\partial}{\partial Z},
$$

such that

$$
\begin{gathered}
n_{1}(x, y) \frac{\partial \Phi_{1}}{\partial x}(x, y)+n_{2}(x, y) \frac{\partial \Phi_{2}}{\partial x}(x, y)=0 ; \\
n_{1}(x, y) \frac{\partial \Phi_{1}}{\partial y}(x, y)+n_{2}(x, y) \frac{\partial \Phi_{2}}{\partial y}(x, y)+n_{3}(x, y)=0 ;
\end{gathered}
$$

(iii) the map $L_{\Phi}: U \rightarrow T_{1} \mathbb{R}^{3}$ defined by $L_{\Phi}(x, y)=\left(\Phi(x, y), v_{\Phi}(x, y)\right)$ is an immersion.

Condition (9) in the definition of normalized Legendrian map germs gives:

Lemma 2.11. For the vector field $v_{\Phi}, n_{1}(0,0) \neq 0$ and $n_{2}(0,0)=n_{3}(0,0)=0$.

By Lemma 2.10(i) and Lemma 2.11, we have the following equality of function germs:

$$
\frac{\partial \Phi_{1}}{\partial x}(x, y)=-\frac{n_{2}(x, y)}{n_{1}(x, y)} \frac{\partial \Phi_{2}}{\partial x}(x, y) .
$$

This, together with condition (8) in the definition of normalized Legendrian maps, implies that

$$
\mathscr{D}(\Phi)(0,0)=(0,0,0) .
$$

The next lemma is clear:

Lemma 2.12. The function germs $n$ and $p$ given by

$$
n(x, y)=-\frac{n_{2}(x, y)}{n_{1}(x, y)} \quad \text { and } \quad p(x, y)=\frac{\partial \Phi_{2}}{\partial x}(x, y)
$$

are of class $C^{\infty}$, and satisfy $\mathscr{D}(\Phi)(x, y)=(n(x, y) p(x, y), p(x, y), y)$.

Lemma 2.13. The function germ $n$ satisfies $n(0,0)=0$ and $\frac{\partial n}{\partial x}(0,0) \neq 0$. 
Proof. By Lemma 2.11, we have $n(0,0)=0$. Assume, for a contradiction, that $\partial n / \partial x$ vanishes at the origin; then so does $\partial n_{2} / \partial x$. At the same time, by differentiating both sides of the equality in Lemma 2.10(ii) with respect to $x$, we have

$$
n_{1}(0,0) \frac{\partial^{2} \Phi_{1}}{\partial x \partial y}(0,0)+\frac{\partial n_{3}}{\partial x}(0,0)=0 .
$$

Because $\Phi$ is a normalized Legendrian map germ such that the origin of $\mathbb{R}^{2}$ is a singular point of $\Phi$, we obtain $\left(\partial n_{3} / \partial x\right)(0,0) \neq 0$, which together with (13) gives

$$
\frac{\partial^{2} \Phi_{1}}{\partial x \partial y}(0,0) \neq 0 \text {. }
$$

From (8), (11), and Lemma 2.11 we have a contradiction.

Definition 2.14. Let $\Phi:\left(\mathbb{R}^{2}, 0\right) \rightarrow\left(\mathbb{R}^{3}, 0\right)$ be a Legendrian map germ, and let $v_{\Phi}$ be a unit normal vector field of $\Phi$ given in the definition of Legendrian map germs. The $C^{\infty}$ function germ $L J_{\Phi}:\left(\mathbb{R}^{2}, 0\right) \rightarrow \mathbb{R}$ defined by

$$
L J_{\Phi}(x, y)=\operatorname{det}\left(\frac{\partial \Phi}{\partial x}(x, y), \frac{\partial \Phi}{\partial y}(x, y), v_{\Phi}(x, y)\right)
$$

is called the Legendrian Jacobian of $\Phi$.

Note that if $v_{\Phi}$ satisfies the conditions of unit normal vector field of $\Phi$, then $-v_{\Phi}$ also satisfies them. Thus, the sign of $L J_{\Phi}(x, y)$ depends on the particular choice of unit normal vector field $v_{\Phi}$. The Legendrian Jacobian of $\Phi$ is also called the signed area density function [Saji et al. 2009b]. Although it is reasonable to call $L J_{\Phi}$ the area density function from the viewpoint of investigating the singular surface $\Phi(U)$ ( $U$ is a sufficiently small neighborhood of the origin of $\mathbb{R}^{2}$ ), it is also reasonable to call it the Legendrian Jacobian from the viewpoint of investigating the singular map germ $\Phi$.

Let $\Phi:\left(\mathbb{R}^{2}, 0\right) \rightarrow\left(\mathbb{R}^{3}, 0\right)$ be a normalized Legendrian map germ and $v_{\Phi}$ a unit normal vector field of $\Phi$. Write

$$
\begin{aligned}
\Phi(x, y) & =\left(\Phi_{1}(x, y), \Phi_{2}(x, y), y\right), \\
v_{\Phi}(x, y) & =n_{1}(x, y) \frac{\partial}{\partial X}+n_{2}(x, y) \frac{\partial}{\partial Y}+n_{3}(x, y) \frac{\partial}{\partial Z} .
\end{aligned}
$$

By Lemma 2.11, we may set

$$
\tilde{v}_{\Phi}(x, y)=\frac{\partial}{\partial X}+\frac{n_{2}(x, y)}{n_{1}(x, y)} \frac{\partial}{\partial Y}+\frac{n_{3}(x, y)}{n_{1}(x, y)} \frac{\partial}{\partial Z} .
$$

We now give a formula for the Legendrian Jacobian. We start with the cross product (vector product)

$$
\frac{\partial \Phi}{\partial x}(x, y) \times \frac{\partial \Phi}{\partial y}(x, y)=\frac{\partial \Phi_{2}}{\partial x}(x, y) \tilde{v}_{\Phi}(x, y) .
$$


This gives

$$
L J_{\Phi}(x, y)=\frac{\left(\partial \Phi_{2} / \partial x\right)(x, y)}{n_{1}(x, y)} .
$$

Proof of Proposition 1.2. (i) Set

$$
\mathscr{I}(\varphi)=\Phi(x, y)=\left(\Phi_{1}(x, y), \Phi_{2}(x, y), y\right) .
$$

By Lemma 2.9, $\Phi$ is a Legendrian map germ. Thus, it is sufficient to show that (8) and (9) in the definition of normalized Legendrian map germs are satisfied.

Set $\varphi(x, y)=(n(x, y) p(x, y), p(x, y), y)$. By the definition of map germs of pedal unfolding type, we have $n(0,0)=0$ and $p(0,0)=0$. It follows that

$$
\frac{\partial \Phi_{2}}{\partial x}(0,0)=p(0,0)=0 .
$$

Thus, condition (8) is satisfied. By Lemma 2.8 , the germ $L_{\Phi}$ given by

$$
L_{\Phi}(x, y)=\left(\Phi(x, y), \frac{\tilde{v}_{\Phi}(x, y)}{\left\|\tilde{v}_{\Phi}(x, y)\right\|}\right)
$$

is a germ of Legendrian lift of $\Phi$, where

$$
\tilde{v}_{\Phi}(x, y)=\frac{\partial}{\partial X}-n(x, y) \frac{\partial}{\partial Y}-\left(\frac{\partial \Phi_{1}}{\partial y}(x, y)-n(x, y) \frac{\partial \Phi_{2}}{\partial y}(x, y)\right) \frac{\partial}{\partial Z} .
$$

Since $n(0,0)=0$ and

$$
\frac{\partial \Phi_{1}}{\partial y}(0,0)=\int_{0}^{0} \frac{\partial n p}{\partial y}(x, 0) d x=0,
$$

we have

$$
\frac{\tilde{v}_{\Phi}(0,0)}{\left\|\tilde{v}_{\Phi}(0,0)\right\|}=\frac{\partial}{\partial X} .
$$

Thus, condition (9) is satisfied, proving part (i) of the proposition.

Proposition 1.2(ii) follows from (12), Lemma 2.12, and Lemma 2.13.

\section{Proof of Theorem 1.3}

Suppose that both $\mathscr{I}: \mathscr{W} \rightarrow \mathscr{Y}$ and $\mathscr{D}: \mathscr{Y} \rightarrow \mathscr{W}$ are well-defined. By the fundamental theorem of calculus, we have $\mathscr{D} \circ \mathscr{I}(\varphi)=\varphi$ for all $\varphi \in \mathscr{W}$, and $\mathscr{I} \circ \mathscr{D}(\Phi)=\Phi$ for all $\Phi \in \mathscr{Y}$. That is, both $\mathscr{I}$ and $\mathscr{D}$ are bijective. Therefore, in order to complete the proof, it is sufficient to show that both $\mathscr{I}$ and $\mathscr{D}$ are well-defined.

Proof that $\mathscr{I}: \mathcal{W} \rightarrow \mathscr{Y}$ is well-defined. Let $\varphi(x, y)=\left(n(x, y) p_{\varphi}(x, y), p_{\varphi}(x, y), y\right)$ be an element of $\mathscr{W}$. Set $\Phi=\mathscr{I}(\varphi)$. Then $\Phi$ is a normalized Legendrian map germ 
by Proposition 1.2. Let $g$ be the Whitney umbrella of pedal unfolding type - see (2) in Section 1:

$$
g(x, y)=\left(x p_{g}(x, y), p_{g}(x, y), y\right)=\left(x\left(x^{2}+y\right),-x^{2}-y, y\right) .
$$

Lemma 3.1. There exists a germ of $C^{\infty}$ diffeomorphism $h:\left(\mathbb{R}^{2}, 0\right) \rightarrow\left(\mathbb{R}^{2}, 0\right)$ such that $h$ has the form $h(x, y)=\left(h_{1}(x, y), h_{2}(y)\right)$ and $p_{\varphi} \circ h(x, y)$ is $x^{2}+y$ or $-\left(x^{2}+y\right)$.

Proof. Since $\varphi$ is a Whitney umbrella of pedal unfolding type, we have

$$
Q\left(p_{\varphi}(x, y), y\right) \cong Q(\varphi) \cong Q(g) \cong Q\left(x^{2}, y\right) .
$$

Thus, we may set $p_{\varphi}(x, 0)=a_{2} x^{2}+o\left(x^{2}\right)\left(a_{2} \neq 0\right)$ by Proposition 2.1. By the Morse lemma with parameters [Bruce and Giblin 1992], there exists a germ of $C^{\infty}$ diffeomorphism $h:\left(\mathbb{R}^{2}, 0\right) \rightarrow\left(\mathbb{R}^{2}, 0\right)$ such that $h$ has the form

$$
h(x, y)=\left(h_{1}(x, y), h_{2}(y)\right) \quad \text { and } \quad p_{\varphi} \circ h(x, y)= \pm\left(x^{2}+q(y)\right)
$$

by a certain $C^{\infty}$ function germ $q:(\mathbb{R}, 0) \rightarrow(\mathbb{R}, 0)$. Since $\varphi$ is $\mathscr{A}$-equivalent to $g$, by Corollary 2.4, $\pm\left(x^{2}+q(y)\right)$ is $\mathscr{K}^{T}$-equivalent to $p_{g}$ and thus $q:(\mathbb{R}, 0) \rightarrow(\mathbb{R}, 0)$ is a germ of $C^{\infty}$ diffeomorphism. Lemma 3.1 follows.

Set $G=\mathscr{I}(g)$. Then, $G$ has the form of (3) from Section 1, which is a normalized swallowtail. Since $G$ is normalized, $\partial / \partial x$ is the null vector field for $G$ defined in [Kokubu et al. 2005; Saji et al. 2009a], that is,

$$
\frac{\partial G}{\partial x}(x, y)=0
$$

holds for any $(x, y)$ which is a singular point of $G$. Since $G$ is a swallowtail, we have, by [Saji et al. 2009a, Corollary 2.5],

$L J_{G}(0,0)=\frac{\partial L J_{G}}{\partial x}(0,0)=0, \quad \frac{\partial^{2} L J_{G}}{\partial x^{2}}(0,0) \neq 0, \quad Q\left(L J_{G}, \frac{\partial L J_{G}}{\partial x}\right) \cong Q(x, y)$.

On the other hand, by (14) and Lemma 3.1, there exists a germ of $C^{\infty}$ diffeomorphism $h:\left(\mathbb{R}^{2}, 0\right) \rightarrow\left(\mathbb{R}^{2}, 0\right)$ and a $C^{\infty}$ function germ $\xi:\left(\mathbb{R}^{2}, 0\right) \rightarrow \mathbb{R}$, such that $h$ has the form $h(x, y)=\left(h_{1}(x, y), h_{2}(y)\right), \xi(0,0) \neq 0$ and we have

$$
L J_{\Phi} \circ h(x, y)=\xi(x, y) L J_{G}(x, y) .
$$

Because $\partial / \partial x$ is the null vector field for $\Phi$ (this is because $\Phi$ is normalized), $L J_{\Phi}$ satisfies

$L J_{\Phi}(0,0)=\frac{\partial L J_{\Phi}}{\partial x}(0,0)=0, \quad \frac{\partial^{2} L J_{\Phi}}{\partial x^{2}}(0,0) \neq 0, \quad Q\left(L J_{\Phi}, \frac{\partial L J_{\Phi}}{\partial x}\right) \cong Q(x, y)$.

Hence, $\Phi$ is a swallowtail by [Saji et al. 2009a, Corollary 2.5], and we have proved that $\mathscr{I}: \mathscr{W} \rightarrow \mathscr{Y}$ is well-defined. 
Proof that $\mathscr{D}: \mathscr{S} \rightarrow \mathscr{W}$ is well-defined.. Let $\Phi$ be an element of $\mathscr{Y}$. Then, by Proposition 2.1, $\mathscr{D}(\Phi)$ is of pedal unfolding type; we must show that it is a Whitney umbrella.

Lemma 3.2. For the Legendrian Jacobian $L J_{\Phi}$, we have $L J_{\Phi}(0,0)=\frac{\partial L J_{\Phi}}{\partial x}(0,0)=0, \quad \frac{\partial^{2} L J_{\Phi}}{\partial x^{2}}(0,0) \neq 0, \quad Q\left(L J_{\Phi}, \frac{\partial L J_{\Phi}}{\partial x}\right) \cong Q(x, y)$.

Proof. Since $\Phi$ is normalized, $\partial / \partial x$ is the null vector field. Since $\Phi$ is a swallowtail, Lemma 3.2 follows from [Saji et al. 2009a, Corollary 2.5].

Since $\mathscr{D}(\Phi)$ is of pedal unfolding type, there exists a $C^{\infty}$ function germ $n$ : $\left(\mathbb{R}^{2}, 0\right) \rightarrow(\mathbb{R}, 0)$ such that

$$
\frac{\partial n}{\partial x}(0,0) \neq 0 \quad \text { and } \quad \frac{\partial \Phi_{1}}{\partial x}(x, y)=n(x, y) \frac{\partial \Phi_{2}}{\partial x}(x, y),
$$

where $\Phi(x, y)=\left(\Phi_{1}(x, y), \Phi_{2}(x, y), y\right)$. Set $p_{\varphi}=\partial \Phi_{2} / \partial x$. By (14), Lemma 3.2, and the Morse lemma with parameters, there is a germ of $C^{\infty}$ diffeomorphism $h:\left(\mathbb{R}^{2}, 0\right) \rightarrow\left(\mathbb{R}^{2}, 0\right)$ such that $h$ has the form $h(x, y)=\left(h_{1}(x, y), h_{2}(y)\right)$ and $p_{\varphi} \circ h(x, y)= \pm\left(x^{2}+y\right)$. Then, by Corollary $2.4, \mathscr{D}(\Phi)$ is $\mathscr{A}$-equivalent to $g$.

\section{Proof of Theorem 1.4}

As with Theorem 1.3, it is sufficient to show that both $\mathscr{I}: \mathcal{N} \rightarrow \mathscr{C}$ and $\mathscr{D}: \mathscr{C} \rightarrow \mathcal{N}$ are well-defined.

Proof that $\mathbb{I}: \mathcal{N} \rightarrow \mathscr{C}$ is well-defined. Let $\varphi(x, y)=\left(n(x, y) p_{\varphi}(x, y), p_{\varphi}(x, y), y\right)$ be an element of $\mathcal{N}$. Set $\Phi=\mathscr{I}(\varphi)$. Then, since $\varphi$ is of pedal unfolding type, $\Phi$ is a normalized Legendrian map germ by Proposition 1.2. Let $g$ be the nonsingular map germ of pedal unfolding type defined by $g(x, y)=\left(x^{2}, x, y\right)$.

Lemma 4.1. There exists a germ of $C^{\infty}$ diffeomorphism $h:\left(\mathbb{R}^{2}, 0\right) \rightarrow\left(\mathbb{R}^{2}, 0\right)$ having the form $h(x, y)=\left(h_{1}(x, y), h_{2}(y)\right)$ and such that $p_{\varphi} \circ h(x, y)=x$.

Proof. Since $\varphi$ is nonsingular and of pedal unfolding type, we have

$$
Q\left(p_{\varphi}(x, y), y\right) \cong Q(\varphi) \cong Q(g) \cong Q(x, y) .
$$

Thus, $\left(p_{\varphi}(x, y), y\right)$ is a germ of $C^{\infty}$ diffeomorphism by Proposition 2.1. From the form of $\left(p_{\varphi}(x, y), y\right)$, its inverse map germ $h:\left(\mathbb{R}^{2}, 0\right) \rightarrow\left(\mathbb{R}^{2}, 0\right)$ has the form $h(x, y)=\left(h_{1}(x, y), h_{2}(y)\right)$. Since $h$ is the inverse map germ of $\left(p_{\varphi}(x, y), y\right)$, it follows that $p_{\varphi} \circ h(x, y)=x$.

Since $\Phi$ is normalized, $\partial / \partial x$ is the null vector field for $\Phi$. By Lemma 4.1 and (14), we have

$$
\frac{\partial L J_{\Phi}}{\partial x}(0,0) \neq 0
$$


Thus, the null vector field $\partial / \partial x$ is transverse to $\left\{(x, y) \mid L J_{\Phi}(x, y)=0\right\}$ at $(0,0) \in$ $\mathbb{R}^{2}$. Hence, $\Phi$ is a cuspidal edge by [Kokubu et al. 2005, Proposition 1.3], showing that $\Phi: \mathcal{N} \rightarrow \mathscr{C}$ is well-defined.

Proof that $\mathscr{D}: \mathscr{C} \rightarrow \mathcal{N}$ is well-defined. Let $\Phi$ be an element of $\mathscr{C}$. By Proposition 1.2, $\mathscr{D}(\Phi)$ is of pedal unfolding type.

Lemma 4.2. The Legendrian Jacobian $L J_{\Phi}$ satisfies

$$
L J_{\Phi}(0,0)=0 \quad \text { and } \quad \frac{\partial L J_{\Phi}}{\partial x}(0,0) \neq 0 .
$$

Proof. Since $\partial / \partial x$ is the null vector field for $\Phi$ and $\Phi$ is a cuspidal edge, Lemma 4.2 follows from [Saji et al. 2009a, Corollary 2.5].

Since $\mathscr{D}(\Phi)$ is of pedal unfolding type, there exists a $C^{\infty}$ function germ $n$ : $\left(\mathbb{R}^{2}, 0\right) \rightarrow(\mathbb{R}, 0)$ such that

$$
\frac{\partial n}{\partial x}(0,0) \neq 0 \quad \text { and } \quad \frac{\partial \Phi_{1}}{\partial x}(x, y)=n(x, y) \frac{\partial \Phi_{2}}{\partial x}(x, y),
$$

where $\Phi(x, y)=\left(\Phi_{1}(x, y), \Phi_{2}(x, y), y\right)$. Set $p_{\varphi}=\partial \Phi_{2} / \partial x$. By Lemma 4.2 and (14), the map germ $(x, y) \mapsto\left(p_{\varphi}(x, y), y\right)$ is a germ of a $C^{\infty}$ diffeomorphism. Thus, $\mathscr{D}(\Phi)$ is nonsingular. Since $\mathscr{D}(\Phi)(0,0)=(0,0,0)$, we have proved that $\mathscr{D}: \mathscr{C} \rightarrow \mathcal{N}$ is well-defined.

\section{Acknowledgement}

The author thanks Kentaro Saji for valuable comments.

\section{References}

[Arnold et al. 1985] V. I. Arnold, S. M. Gusein-Zade, and A. N. Varchenko, Singularities of differentiable maps, vol. 1, Monographs in Mathematics 82, Birkhäuser, Boston, 1985. MR 86f:58018 Zbl 0554.58001

[Bruce and Giblin 1992] J. W. Bruce and P. J. Giblin, Curves and singularities, 2nd ed., Cambridge University Press, 1992. MR 93k:58020 Zbl 0770.53002

[Izumiya 1987] S. Izumiya, "On Legendrian singularities", Proc. Amer. Math. Soc. 101:4 (1987), 748-752. MR 89c:58016 Zbl 0639.58006

[Kokubu et al. 2005] M. Kokubu, W. Rossman, K. Saji, M. Umehara, and K. Yamada, "Singularities of flat fronts in hyperbolic space", Pacific J. Math. 221:2 (2005), 303-351. MR 2006k:53102 Zbl 1110.53044

[Mather 1969] J. N. Mather, "Stability of $C^{\infty}$ mappings, IV: Classification of stable germs by $R$-algebras", Inst. Hautes Études Sci. Publ. Math. 37 (1969), 223-248. MR 43 \#1215b Zbl 0202.55102

[Mond 1985] D. Mond, "On the classification of germs of maps from $\mathbf{R}^{2}$ to $\mathbf{R}^{3}$ ", Proc. London Math. Soc. (3) 50:2 (1985), 333-369. MR 86g:58021 Zbl 0557.58006

[Nishimura 2010] T. Nishimura, "Singularities of one-parameter pedal unfoldings of spherical pedal curves", J. Singul. 2 (2010), 160-169. MR 2763024 
[Saji et al. 2009a] K. Saji, M. Umehara, and K. Yamada, " $A_{k}$ singularities of wave fronts", Math. Proc. Cambridge Philos. Soc. 146:3 (2009), 731-746. MR 2010h:58060

[Saji et al. 2009b] K. Saji, M. Umehara, and K. Yamada, "The geometry of fronts", Ann. of Math.

(2) 169:2 (2009), 491-529. MR 2010e:58042 Zbl 1177.53014

[Whitney 1943] H. Whitney, "The general type of singularity of a set of $2 n-1$ smooth functions of $n$ variables", Duke Math. J. 10 (1943), 161-172. MR 4,193b Zbl 0061.37207

[Whitney 1944] H. Whitney, "The singularities of a smooth $n$-manifold in ( $2 n-1)$-space", Ann. of Math. (2) 45 (1944), 247-293. MR 5,274a Zbl 0063.08238

[Zakalyukin 1976] V. Zakalyukin, "Lagrangian and Legendrian singularities", Funktsional. Anal. $i$ Prilozhen. 10:1 (1976), 26-36. In Russian; translated in Funct. Anal. Appl. 10:1 (1976), 23-31. Zbl 0331.58007

[Zakalyukin 1983] V. M. Zakalyukin, "Reconstructions of fronts and caustics depending on a parameter, and versality of mappings", pp. 56-93 in Современные проблемы математики, vol. 22, edited by R. V. Gamkrelidze, Akad. Nauk SSSR Vsesoyuz. Inst. Nauchn. i Tekhn. Inform., Moscow, 1983. In Russian; translated in J. Sov. Math. 27 (1984), 2713-2735. MR 85h:58029 Zbl 0534.58014

Received July 30, 2011.

TAKASHI NISHIMURA

Group of MATHEMATICAL SCIENCES

RESEARCH INSTITUTE OF ENVIRONMENT AND INFORMATION SCIENCES

YOKOHAMA NATIONAL UNIVERSITY

YOKOHАMA 240-8501

JAPAN

nishimura-takashi-yx @ynu.jp 


\title{
PACIFIC JOURNAL OF MATHEMATICS
}

\author{
http://www.pjmath.org \\ Founded in 1951 by
}

E. F. Beckenbach (1906-1982) and F. Wolf (1904-1989)

\section{EDITORS}

V. S. Varadarajan (Managing Editor)

Department of Mathematics

University of California

Los Angeles, CA 90095-1555

pacific@math.ucla.edu

Vyjayanthi Chari

Department of Mathematics

University of California

Riverside, CA 92521-0135

chari@math.ucr.edu

\section{Robert Finn}

Department of Mathematics Stanford University

Stanford, CA 94305-2125

finn@math.stanford.edu

Kefeng Liu

Department of Mathematics

University of California

Los Angeles, CA 90095-1555

liu@math.ucla.edu
Darren Long

Department of Mathematics

University of California

Santa Barbara, CA 93106-3080

long@math.ucsb.edu

Jiang-Hua Lu

Department of Mathematics

The University of Hong Kong

Pokfulam Rd., Hong Kong jhlu@maths.hku.hk

Alexander Merkurjev

Department of Mathematics University of California

Los Angeles, CA 90095-1555 merkurev@math.ucla.edu
Sorin Popa

Department of Mathematics

University of California

Los Angeles, CA 90095-1555

popa@math.ucla.edu

Jie Qing

Department of Mathematics

University of California

Santa Cruz, CA 95064

qing@ cats.ucsc.edu

Jonathan Rogawski

Department of Mathematics

University of California

Los Angeles, CA 90095-1555

jonr@math.ucla.edu

\section{PRODUCTION}

pacific@math.berkeley.edu

Silvio Levy, Scientific Editor Matthew Cargo, Senior Production Editor

ACADEMIA SINICA, TAIPEI

CALIFORNIA INST. OF TECHNOLOGY

INST. DE MATEMÁTICA PURA E APLICADA

KEIO UNIVERSITY

MATH. SCIENCES RESEARCH INSTITUTE

NEW MEXICO STATE UNIV.

OREGON STATE UNIV.

\section{SUPPORTING INSTITUTIONS}

STANFORD UNIVERSITY
UNIV. OF BRITISH COLUMBIA
UNIV. OF CALIFORNIA, BERKELEY
UNIV. OF CALIFORNIA, DAVIS
UNIV. OF CALIFORNIA, LOS ANGELES
UNIV. OF CALIFORNIA, RIVERSIDE
UNIV. OF CALIFORNIA, SAN DIEGO
UNIV. OF CALIF., SANTA BARBARA

UNIV. OF CALIF., SANTA CRUZ

UNIV. OF MONTANA

UNIV. OF OREGON

UNIV. OF SOUTHERN CALIFORNIA

UNIV. OF UTAH

UNIV. OF WASHINGTON

WASHINGTON STATE UNIVERSITY

These supporting institutions contribute to the cost of publication of this Journal, but they are not owners or publishers and have no responsibility for its contents or policies.

See inside back cover or www.pjmath.org for submission instructions.

The subscription price for 2011 is US \$420/year for the electronic version, and \$485/year for print and electronic.

Subscriptions, requests for back issues from the last three years and changes of subscribers address should be sent to Pacific Journal of Mathematics, P.O. Box 4163, Berkeley, CA 94704-0163, U.S.A. Prior back issues are obtainable from Periodicals Service Company, 11 Main Street, Germantown, NY 12526-5635. The Pacific Journal of Mathematics is indexed by Mathematical Reviews, Zentralblatt MATH, PASCAL CNRS Index, Referativnyi Zhurnal, Current Mathematical Publications and the Science Citation Index.

The Pacific Journal of Mathematics (ISSN 0030-8730) at the University of California, c/o Department of Mathematics, 969 Evans Hall, Berkeley, CA 94720-3840, is published monthly except July and August. Periodical rate postage paid at Berkeley, CA 94704, and additional mailing offices. POSTMASTER: send address changes to Pacific Journal of Mathematics, P.O. Box 4163, Berkeley, CA 94704-0163.

PJM peer review and production are managed by EditFLOW ${ }^{\mathrm{TM}}$ from Mathematical Sciences Publishers.

PUBLISHED BY PACIFIC JOURNAL OF MATHEMATICS

at the University of California, Berkeley 94720-3840

A NON-PROFIT CORPORATION

Typeset in IATEX

Copyright $(2011$ by Pacific Journal of Mathematics 


\title{
PACIFIC JOURNAL OF MATHEMATICS
}

\author{
Volume $252 \quad$ No. $2 \quad$ August 2011
}

Remarks on a Künneth formula for foliated de Rham cohomology

MÉLANIE BERTELSON

$K$-groups of the quantum homogeneous space $\mathrm{SU}_{q}(n) / \mathrm{SU}_{q}(n-2)$

275

PARTHA SARATHI CHAKRABORTY and S. SUNDAR

A class of irreducible integrable modules for the extended baby TKK algebra

293

XUEWU CHANG and SHaOBin TAN

Duality properties for quantum groups

SOPHIE CHEMLA

Representations of the category of modules over pointed Hopf algebras over $\mathbb{S}_{3}$ and $\mathbb{S}_{4}$

Agustín García IGLESIAS and Martín Mombelli

$(p, p)$-Galois representations attached to automorphic forms on $\mathrm{GL}_{n}$

EKNATH GHATE and NARASIMHA KUMAR

On intrinsically knotted or completely 3-linked graphs 407

Ryo Hanaki, Ryo NikKUni, Kouki TaNiYama and AKIKo YAMAZAKI

Connection relations and expansions

Mourad E. H. ISMAIL and Mizan Rahman

Characterizing almost Prüfer $v$-multiplication domains in pullbacks

QING LI

Whitney umbrellas and swallowtails

TAKASHI NISHIMURA

The Koszul property as a topological invariant and measure of singularities

HAL SADOFSKY and BRAD SHELTON

A completely positive map associated with a positive map

ERLING STøRMER

Classification of embedded projective manifolds swept out by rational homogeneous varieties of codimension one

KiWAMU Watanabe

Note on the relations in the tautological ring of $\mu_{g}$ 